\title{
DEVELOPMENT AND VALIDATION OF RP-HPLC METHOD FOR THE ESTIMATION OF ESCITALOPRAM OXALATE AND FLUPENTIXOL DIHYDROCHLORIDE IN COMBINED DOSAGE FORM AND PLASMA
}

\author{
MALATHI SELLAPPAN ${ }^{*}$, DARTHI DEVAKUMAR²
}

1,2* Department of Pharmaceutical Analysis, PSG College of Pharmacy, Peelamedu, Coimbatore, Tamilnadu, 641004, India

Email: malathisanju@gmail.com

Received: 05 Oct 2018, Revised and Accepted: 12 Dec 2020

\begin{abstract}
Objective: The objective of the study was to develop a simple and rapid chromatographic method for quantification of escitalopram oxalate and flupentixol dihydrochloride in combined dosage form and plasma.

Methods: The separation was achieved with a sun fire $\mathrm{C}_{8}[150 \times 4.6 \mathrm{~mm}] 3.5 \mu \mathrm{m}$ column with an isocratic mobile phase containing a mixture of potassium dihydrogen orthophosphate buffer: methanol: acetonitrile [30:60:10 v/v/v] pH adjusted to 11 . The flow rate of the mobile phase was $1.5 \mathrm{ml} / \mathrm{min}$ with a Photodiode array [PDA] detection at $230 \mathrm{~nm}$.

Results: The HPLC method was developed and validated with respective linearity, accuracy, and precision, detection of limit, robustness, and specificity. The precision of the results stated as the relative standard deviation was below $2 \%$. The calibration curve was linear over a concentration range from 10-50 $\mu \mathrm{g} / \mathrm{ml}$ for escitalopram oxalate and $1-5 \mu \mathrm{g} / \mathrm{ml}$ for flupentixol dihydrochloride with a correlation co-efficient 0.994 and 0.977 respectively. The accuracy of the method was demonstrated at levels in the range of $100 \%$ and $120 \%$ of the specification limit. The recovery of escitalopram oxalate and flupentixol dihydrochloride was found to be in the range of $90 \%$ to $88 \%$, respectively. The lowest detection limits were found to be $2 \mu \mathrm{g} / \mathrm{ml}$ for escitalopram oxalate and $0.1 \mu \mathrm{g} / \mathrm{ml}$ for flupentixol dihydrochloride. The lowest quantification limits were found to be $5 \mu \mathrm{g} / \mathrm{ml}$ of escitalopram oxalate and $0.5 \mu \mathrm{g} / \mathrm{ml}$ of flupentixol dihydrochloride.
\end{abstract}

Conclusion: The developed method was validated for linearity, accuracy, precision, the limit of detection and quantification, specificity. The method was applied successfully for the determination of escitalopram oxalate and flupentixol dihydrochloride in the combined dosage form and plasma.

Keywords: Escitalopram oxalate, Flupentixol dihydrochloride, High-performance liquid chromatography, Human plasma, Validation

(c) 2021 The Authors. Published by Innovare Academic Sciences Pvt Ltd. This is an open access article under the CC BY license (https://creativecommons.org/licenses/by/4.0/) DOI: https://dx.doi.org/10.22159/ijpps.2021v13i2.30158. Journal homepage: https://innovareacademics.in/journals/index.php/ijpps.

\section{INTRODUCTION}

Depression is a mood disorder that causes a persistent feeling of sadness and loss of interest, also called a major depressive disorder or clinical depression, it affects feel, think, and behave and can lead to a variety of emotional and physical problems. Escitalopram oxalate $[E S C]$ is an orally administered selective serotonin reuptake inhibitor. It is the pure $\mathrm{S}$ enantiomers of racemic bicyclic phthalate derivative of citalopram. Chemically it is S-1-[3-Dimethylamino]propyl]-1-[4-flurophenyl]-1, dihydro isobenzofuran-5-carbonitrile [1]. ESC is used to treat depression and anxiety. It works by helping to restore the balance of a certain natural substance [serotonin] in the brain. ESC belongs to a class of drugs known as Selective Serotonin Reuptake Inhibitors [SSRI]. It may improve your energy level and feelings of well-being and decrease nervousness. Flupentixol dihydrochloride [FLU] is an antipsychotic narcoleptic drug. It is a thioxanthene and therefore closely related to the phenothiazines. Chemically it is [EZ]-2-[4-[3-[2-[trifluoro-methyl] thioxanthen-9-ylidene] Propyl] piperazin-1-yl] ethanol dihydrochloride [2]. Its primary use is as a long-acting injection given two or three weekly to people with schizophrenia who have poor compliance with medication and suffer frequent relapses of illness. It is a D1 and D2 receptor antagonist. A Literature survey revealed that several methods are developed for ESC and FLU individually [3-10]. Liquid chromatographic methods and UV first derivative methods have been developed for ESC and FLU in pharmaceutical dosage form [11-14]. To the best of our knowledge, no information related to the high-performance liquid chromatography determination of ESC and FLU in pharmaceutical dosage form and plasma has ever been mentioned in the literature. So the present study was aimed to develop a precise, rapid analytical method for estimation of ESC and FLU in combined dosage form and plasma. The developed method was validated according to the ICH guidelines [15].

\section{MATERIALS AND METHODS}

\section{Instruments}

The chromatography analysis was performed using a Shimadzu HPLC module Shimadzu SPD-M10 AVP, Japan equipped with a PDA detector, gradient solvent manager, and manual sample system. The $\mathrm{pH}$ of the solutions was measured by $\mathrm{pH}$ meter Lab India, India.

\section{Chemicals and reagent}

Shangri-La industries supplied a sample of ESC and FLU. Methanol HPLC grade and potassium dihydrogen orthophosphate were purchased in himedia laboratory pvt Ltd, Mumbai. Water for HPLC was purchased in thermo fisher scientific, Mumbai. Acetonitrile HPLC grade was purchased in sigma Aldrich chemicals pvt Ltd, Bangalore. Ammonia solution was purchased in Loba chem. Mumbai.

Chromatographic conditions

The method was developed by using sun fire $C_{8}[150 \times 4.6 \mathrm{~mm}] 3.5$ $\mu \mathrm{m}$ column with an isocratic mobile phase containing a mixture of potassium dihydrogen orthophosphate: methanol: acetonitrile [30:60:10 v/v/v]. The $\mathrm{pH}$ was adjusted to 11 . The Flow rate of the mobile phase was $1.5 \mathrm{ml} / \mathrm{min}$. The eluted compounds were monitored at a wavelength of $230 \mathrm{~nm}$ [11].

Preparation of diluents

Methanol was used as diluents for this experiment.

\section{Preparation of the mobile phase}

A mixture of potassium dihydrogen orthophosphate, methanol and acetonitrile in the ratio of $30: 60: 10[\mathrm{v} / \mathrm{v} / \mathrm{v}]$ was prepared and the $\mathrm{pH}$ adjusted to 11 with $30 \%$ ammonia solution. 


\section{Preparation of standard solution}

A Combined standard stock solution containing $1000 \mu \mathrm{g} / \mathrm{ml}$ of ESC and FLU was prepared in methanol. The standard working solution of ESC and FLU were prepared by serial dilution of the stock solution. This stock solution was diluted appropriately to prepare the standard of ESC was 10-50 $\mu \mathrm{g} / \mathrm{ml}$ and FLU was 1-5 $\mu \mathrm{g} / \mathrm{ml}$, respectively [13].

\section{Preparation of sample solution}

The sample solution was prepared by taking ten tablets containing 5 $\mathrm{mg}$ of ESC and $0.5 \mathrm{mg}$ of FLU were weighed; the average weight was determined and ground to a fine powder using a glass mortar and pestle. An equivalent of $10 \mathrm{mg}$ ESC and $1 \mathrm{mg}$ of FLU was accurately weighed and transferred to a separate $100 \mathrm{ml}$ volumetric flask, The volume was adjusted with methanol and the resultant solution was sonicated for $15 \mathrm{~min}$. This solution was then filtered through Whatmann filter paper. Further dilutions were made from this stock solution to get the required concentration [13].

\section{Validation procedure}

Method validation was achieved as per ICH guidelines for the determination of ESC and FLU. The following validation features were addressed linearity, detection limit, quantification limit, precision, accuracy, robustness, and specificity [15].

\section{Linearity}

Standard solutions at five concentrations ranging from ESC 10-15 $\mu \mathrm{g} / \mathrm{ml}$ and FLU $1-5 \mu \mathrm{g} / \mathrm{ml}$ were prepared and analyzed to demonstrate the linearity. The regression curve was obtained by plotting peak area versus concentration. The regression equation was obtained by using the least-squares method.

\section{Limits of detection and quantification [LOD and LOQ]}

The sensitivity of the method was measured by calculating the limit of detection and limit of quantification. LOD and LOQ were determined by injecting decreasing amounts of drugs. The lowest concentration at which peak is determined is called LOD was found out to be $2 \mu \mathrm{g} / \mathrm{ml}$ for ESC and $0.1 \mu \mathrm{g} / \mathrm{ml}$ for FLU. The lowest concentration at which peak can be quantified is called LOQ was found be for $5 \mu \mathrm{g} / \mathrm{ml} \mathrm{ESC}$ and $0.5 \mu \mathrm{g} / \mathrm{ml}$ for FLU.

\section{Accuracy}

The standard addition and recovery experiments were conducted to demonstrate the accuracy of the method. The accuracy of the method evaluated in triplicate at two concentration levels $100 \%$ and $120 \%$ of target test concentration and the percentages of recoveries were calculated.

\section{System suitability criteria}

The system suitability test was well defined based on the result found in several results chromatograms. The column efficiency was determined from the analyte peak; the tailing factor was below 2 . All the system suitability criteria during the validation of the study and batch analysis study within the acceptance limit. The results of system suitability depicted in table 1.

Table 1: System suitability data

\begin{tabular}{lll}
\hline Peak & ESC & FLU \\
\hline Retention time & $2.1 \min$ & $4.8 \mathrm{~min}$ \\
Peak area & 151325 & 28400 \\
USP plate count & 4123.1 & 3998.4 \\
USP tailing & 1.3 & 1.6 \\
\hline
\end{tabular}

\section{Preparation of standard solution}

The developed method was utilized for the estimation of ESC and FLU in plasma. A stock solution was prepared by dissolving accurately weighed $10 \mathrm{mg}$ of ESC and $1 \mathrm{mg}$ of FLU in $10 \mathrm{ml}$ of methanol to yield a concentration of $1000 \mu \mathrm{g} / \mathrm{ml}$ of ESC and 100 $\mu \mathrm{g} / \mathrm{ml}$ of FLU, respectively. The solution was sonicated for $20 \mathrm{~min}$ serious of aliquots of drug solution containing $10-50 \mu \mathrm{g} / \mathrm{ml}$ of ESC and $1-5 \mu \mathrm{g} / \mathrm{ml}$ of FLU were prepared. To each of the five centrifuge tubes, $0.5 \mathrm{ml}$ of the above-prepared solutions were transferred. To this $0.5 \mathrm{ml}$ of plasma was added and $1 \mathrm{ml}$ of extracting solvent of methanol was added to the tube and centrifuged for $20 \mathrm{~min}$ at 3000 Revolution per minute [RPM]. Further supernatant liquids were collected in another eppendorf tube and the supernatant was injected into the analytical column.

\section{Preparation of sample solution}

A Fixed-dose working sample was prepared according to the dosage form of rexipra $\mathrm{fx}$ 5tablets. Accurately weighed samples of rexipra fx 5 contains 5: $0.5 \mathrm{mg}$ of ESC and FLU were transferred into a $10 \mathrm{ml}$ volumetric flask, dissolved in methanol and sonicated for $20 \mathrm{~min}$ and filtered the solution and from that aliquots solution are prepared. From the above-prepared sample solution [ESC $10-50 \mu \mathrm{g} / \mathrm{ml}$ and FLU $1-5 \mu \mathrm{g} / \mathrm{ml}], 0.5 \mathrm{ml}$ was pipette out and spiked into $0.5 \mathrm{ml}$ of plasma in a polypropylene tube [Torson's] then the tube was cyclomixed for 5 min. Then the $1 \mathrm{ml}$ of methanol was added to the tube and centrifuged for $20 \mathrm{~min}$ at $3000 \mathrm{rpm}$. Further supernatant liquids were collected in another eppendorf tube and the supernatant was injected into the analytical column. The calibration curve was plotted using the peak area of standard drugs Vs concentration of the standard solution. The peak area of the sample chromatogram was compared and the amount of ESC and FLU was calculated.

\section{Extracting procedure}

ESC $10-50 \mu \mathrm{g} / \mathrm{ml}$ and $1-5 \mu \mathrm{g} / \mathrm{ml}$ of FLU, $0.5 \mathrm{ml}$ was pipette out and spiked into $0.5 \mathrm{ml}$ of plasma in a polypropylene tube [Torson's].
Then the tube was cyclomixed for $5 \mathrm{~min}$. Then the $1 \mathrm{ml}$ of methanol was added to the tube and centrifuged for $20 \mathrm{~min}$ at $3000 \mathrm{RPM}$. Further supernatant liquids were collected in another eppendorf tube and the supernatant was injected into the analytical column.

\section{Chromatographic conditions}

The drug was estimated at $230 \mathrm{~nm}$ using a photodiode array detector to maximize the signal of the compound and minimize the signal of plasma interferents. The chromatographic separation was performed on a $\mathrm{C}_{8}$ column [ $150 \mathrm{~mm}$ length $\times 4.6 \mathrm{~mm}$ diameter, $5 \mu \mathrm{m}$ particle size] Sunfire column. The mobile phase consists of potassium dihydrogen orthophosphate: methanol: acetonitrile [30:60:10] pH adjusted to 11 . The mobile phase is pumped at a flow rate of $1.5 \mathrm{ml} / \mathrm{min}$. The mobile phase was sonicated for $15 \mathrm{~min}$ using an ultrasonicator. The developed HPLC method was optimized by monitoring chromatographic parameters, including retention time, column efficiency of the several compositions, and flow rate of the mobile phase.

\section{Validation}

The suggested analytical method was validated according to ICH guidelines for certain parameters such as selectivity, linearity, accuracy, LOD, LOQ and precision. Methanol was used as an extraction solvent as the efficiency of the extraction was more with methanol than with other solvents.

\section{Linearity}

To find out the linearity range of the proposed HPLC method in plasma, curves were constructed by plotting peak areas obtained for the analyte against their concentrations. A good linear relationship $\left[\mathrm{R}^{2}=0.99\right]$ was observed between the concentration of ESC and FLU and their corresponding peak areas. The relevant regression equations were $Y=9588 x+44345$ and $Y=15659 X+14881$ [where $Y$ is the peak area and $X$ is the concentration of ESC and FLU $[\mu \mathrm{g} / \mathrm{ml}]$. The slope, intercept, and correlation coefficient of the plots for ESC was 
9588, 44345 and 0.995 . The slope, intercept, and correlation coefficient of the plots for the FLU was 15659,14881 and 0.970 . The linearity range for ESC and FLU and their corresponding graphs are shown in fig. 1 and 2.

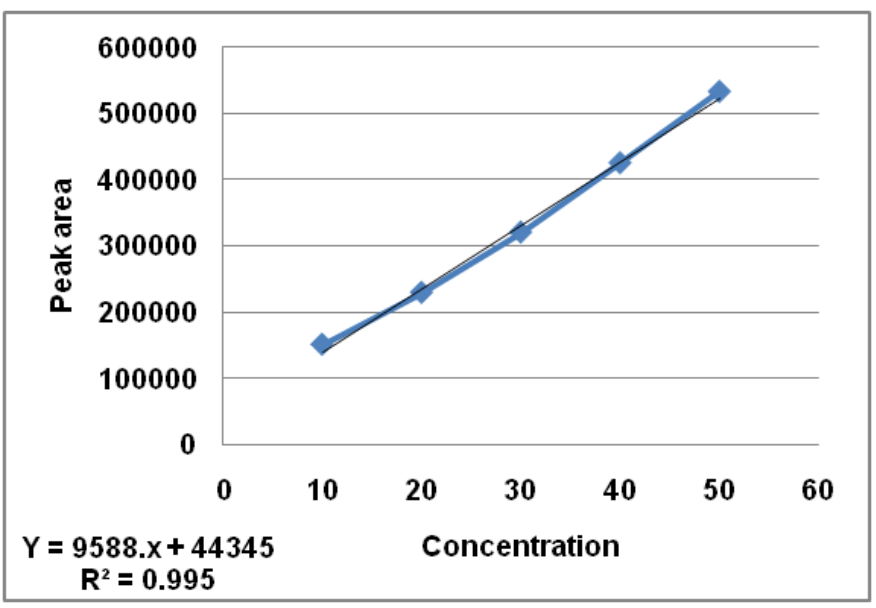

Fig. 1: Linearity graph of ESC

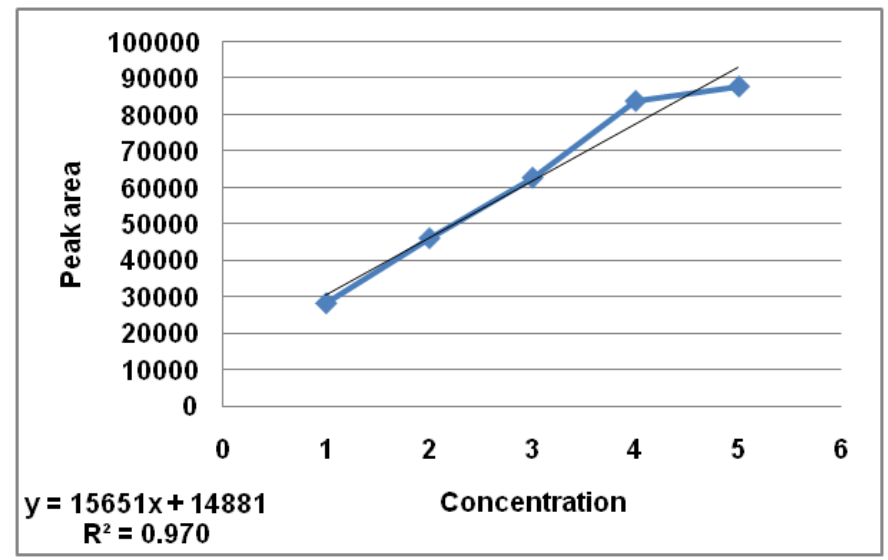

Fig. 2: Linearity graph of FLU

\section{Precision}

The Precision of the method was ascertained in terms of repeatability, intraday and interday precision. Repeatability was determined by injecting $30 \mu \mathrm{g} / \mathrm{ml}$ of ESC and $3 \mu \mathrm{g} / \mathrm{ml}$ of FLU in six replicates and respective areas were calculated. For intra-day and inter-day variations, three concentrations of 20,30 and $40 \mu \mathrm{g} / \mathrm{ml}$ for ESC and 2, 3 and $4 \mu \mathrm{g} / \mathrm{ml}$ for FLU were selected from the linearity range. Intraday analysis was carried on the same day in three replicates. The interday analysis was carried on three different days in three replicates. The precision of the proposed method i.e., the intra and inter-day variations in the peak areas of the drugs solutions in plasma was calculated in terms of \% Relative standard deviation [RSD] and the results are represented in tables 3,4 and 5 . Statistical evaluation revealed that the $\%$ RSD of the drugs at linearity levels for 6 injections was less than 2.0.

\section{Limit of detection and quantification [LOD and LOQ]}

LOD for ESC and FLU in plasma were found to be $2 \mu \mathrm{g} / \mathrm{ml}$ and $0.1 \mu \mathrm{g} / \mathrm{ml}$, respectively. The LOQ for ESC and FLU in plasma was found to be $5 \mu \mathrm{g} / \mathrm{ml}$ and $0.5 \mu \mathrm{g} / \mathrm{ml}$, respectively.

\section{Peak purity}

A Peak purity test was performed for the standard drug peaks. Peak purity index values of ESC and FLU were found to be 0.999, respectively. Values close to one indicates the purity of the peaks.

\section{RESULTS AND DISCUSSION}

Chromatogram depicting the method development of ESC and FLU

A novel, precise and bioanalytical method was developed for the simultaneous estimation of ESC and FLU in plasma samples using sun fire $C_{8}[150 \times 4.6 \mathrm{~mm}] 3.5 \mu \mathrm{m}$ column with a mobile phase containing a mixture of potassium dihydrogen orthophosphate buffer: methanol: acetonitrile [30:60:10 v/v/v] pH adjusted to 11 . The flow rate of the mobile phase was $1.5 \mathrm{ml} / \mathrm{min}$ with [PDA] detection at $230 \mathrm{~nm}$. The chromatogram [fig. 3] obtain from method 1 was found to be no FLU peak; only ESC gave a single sharp peak. The chromatogram [fig. 4] attain for method 2 was found to be no ESC and FLU peak. The chromatogram [fig. 5] accessed for method 3 was found to be no ESC peak; only FLU gave a peak. From the above methods, ESC gave a peak with methanol and FLU gave a peak with acetonitrile. Accordingly, further trials were performed by altering the mobile phase system. The chromatogram [fig. 6] gathered for method 4 were found to be good peak shape, but it was not properly separated. Finally, the chromatogram [fig. 7] the method 5 was found to be perfect to eluted peak with excellent peak shape the tailing factor and retention time were observe within the limit; therefore, the mobile phase ratio was fixed at methanol: acetonitrile: buffer [60:10:30] pH 11 with $1.5 \mathrm{ml} / \mathrm{min}$ flow rate. The retention time for ESC and FLU was 2.1 and 4.8 , respectively. 


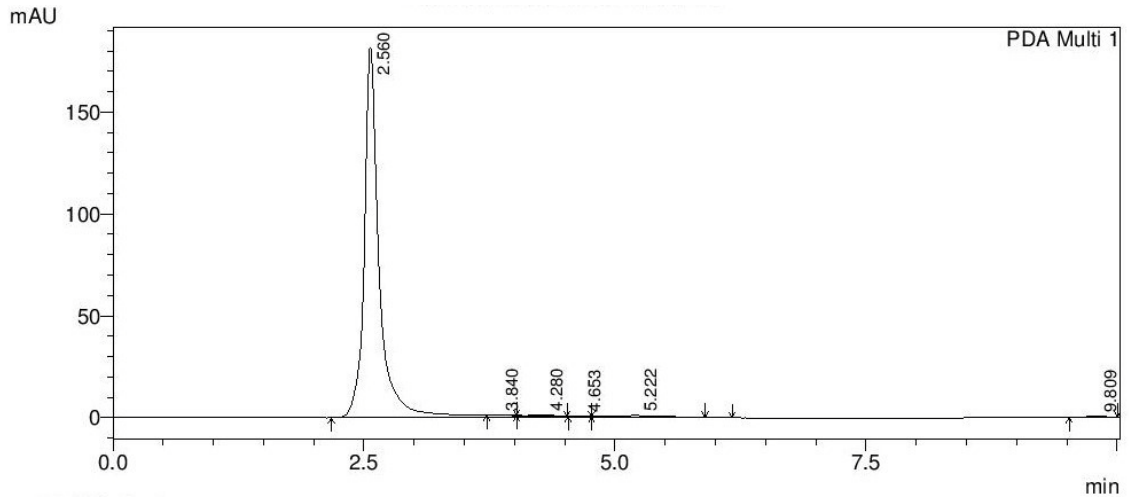

Fig. 3: HPLC chromatogram for method 1

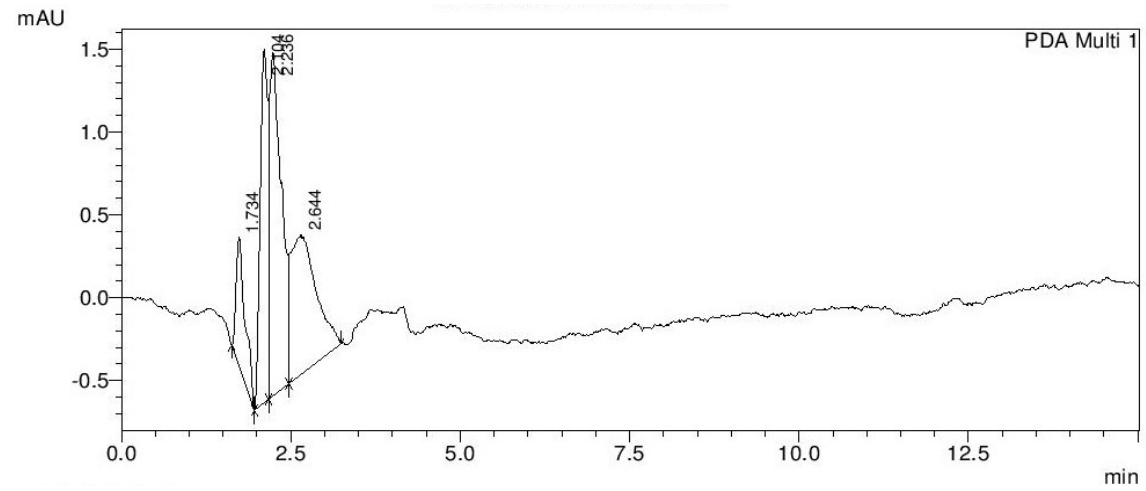

Fig. 4: HPLC chromatogram for method 2

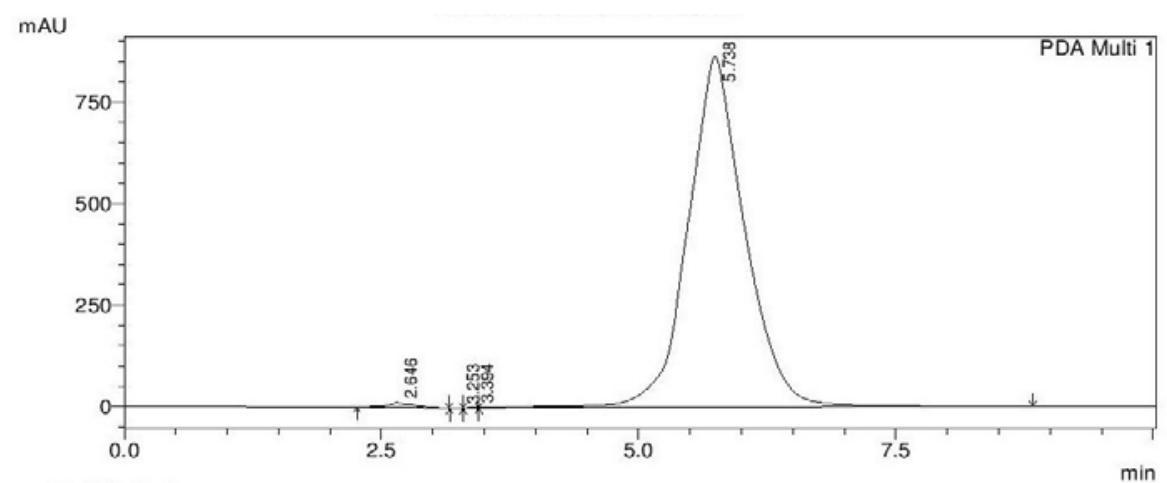

Fig. 5: HPLC chromatogram for method 3

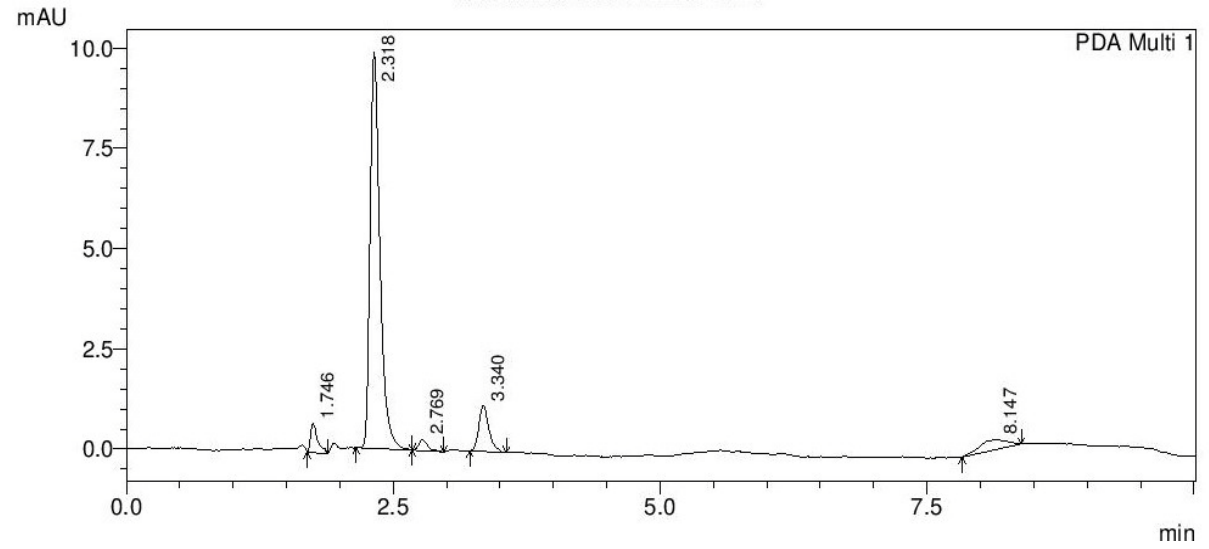

Fig. 6: HPLC chromatogram for method 4 
mAU

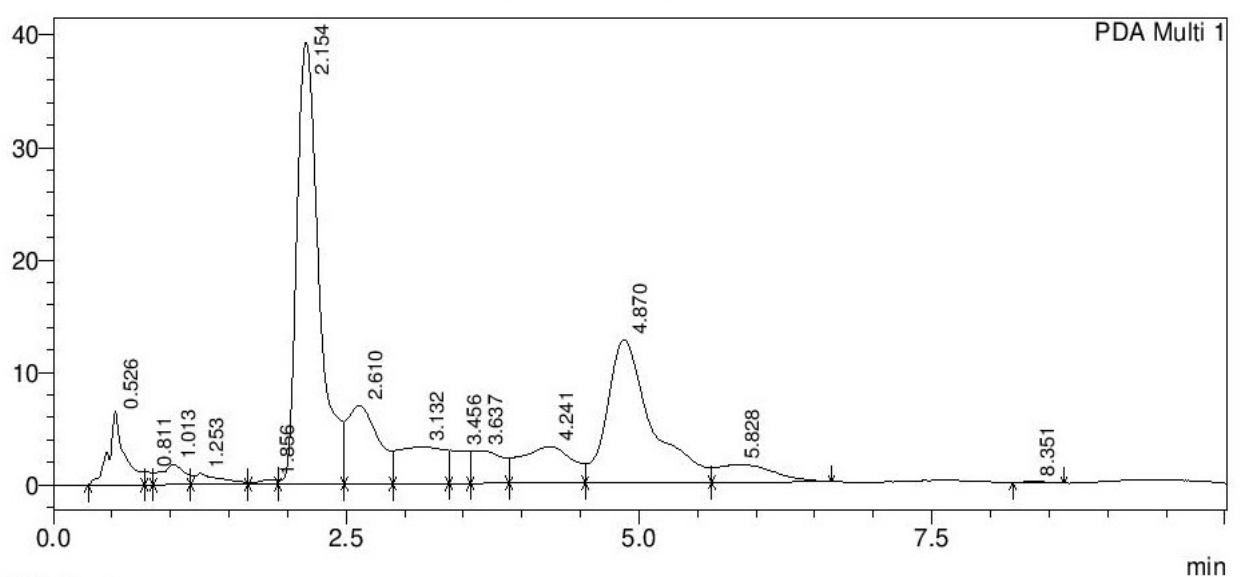

Fig. 7: HPLC chromatogram for method 5

A Literature survey revealed that several methods were developed for ESC and FLU individually [3-10]. UV first derivative method has been developed for ESC and FLU in pharmaceutical dosage form [14]. There are some HPLC methods [11-13] reported in the literature, but these methods are developed only for routine analysis of ESC and FLU in bulk and formulation studies, not for plasma. No information related to the high-performance liquid chromatography determination of ESC and FLU in plasma has ever been mentioned in the literature. The developed HPLC method was utilized for the estimation of the combined drugs by in vitro method. Different extractions were tried using acetonitrile, methanol, and dimethylformamide. Extraction efficiency was good in methanol. So methanol was selected as the solvent. Retention time of ESC and FLU were found to be $2.0 \mathrm{~min}$ and $4.3 \mathrm{~min}$, respectively. The method highlights the importance of the liquid phase extracting technique in bio-analytical sample preparation. Calibration curve was plotted using peak area of standard drugs Vs concentration of the standard solution. The relevant regression equations were 0.994 for ESC and 0.977 for FLU. The inter-day and intra-day variations in plasma by the proposed method showed percentage relative standard deviation were less than $2 \%$, indicating that the method is precise.

\section{Accuracy}

Accuracy of the method was determined by replicates [ $\mathrm{n}=2]$ analysis, carried out using three solutions prepared by standard addition of pure active pharmaceutical ingredients at two different concentration levels $100 \%$ and $120 \%$. Accuracy was calculated by comparing the difference between the spiked value [theoretical value] and that actually found value. Results are presented in the term of \% recovery of the active pharmaceutical ingredients. The results are shown in table 2.

Table 2: Accuracy

\begin{tabular}{llllll}
\hline S. No. & Level & \% Recovery & & \% RSD* & \\
\cline { 3 - 6 } & & ESC & FLU & ESC & FLU \\
\hline 1. & $100 \%$ & 90.2 & 89.7 & 0.82 & 0.71 \\
2. & $120 \%$ & 88.3 & 85.3 & 0.63 & 0.94 \\
\hline
\end{tabular}

*Relative standard deviations

Table 3: Interday precision

\begin{tabular}{lllll}
\hline S. No. & Drug name & Concentration $[\boldsymbol{\mu g} / \mathbf{m l}]$ & {$[$ mean \pm SD] } & \% RSD* \\
\hline 1. & ESC & 20 & $391260.7 \pm 5894.9$ & 1.5 \\
2. & FLU & 2 & $245287.7 \pm 2066.3$ & 0.84 \\
\hline
\end{tabular}

*Relative standard deviations [n=3] SD = Standard deviation, $n=$ number of injection

Table 4: Intraday precision

\begin{tabular}{lllll}
\hline S. No. & Drug name & Concentration $[\boldsymbol{\mu g} / \mathbf{m l}]$ & [mean \pm SD] & \% RSD $^{*}$ \\
\hline 1. & ESC & 20 & $286000 \pm 5993.8$ & 2.09 \\
2. & FLU & 2 & $214557.7 \pm 3358.1$ & 1.56 \\
\hline
\end{tabular}

*Relative standard deviations [n=3] SD = Standard deviation, $n=$ number of injection

Table 5: Repeatability

\begin{tabular}{lllll}
\hline S. No. & Drug name & Concentration $[\boldsymbol{\mu g} / \mathbf{m l}]$ & [mean \pm SD] & \% RSD* \\
\hline 1. & ESC & 30 & $402716.7 \pm 7198.3$ & 1.78 \\
2. & FLU & 3 & $298276.3 \pm 4579.2$ & 1.53 \\
\hline
\end{tabular}

*Relative standard deviations [ $\mathrm{n}=3$ ] SD = Standard deviation, $\mathrm{n}=$ number of injection 
Significant percentage of recovery was observed, which shows the extraction efficiency of the method. The chromatogram for combination drugs in plasma was shown in [fig. 8]. Extraction efficiency was determined by significant recovery studies.

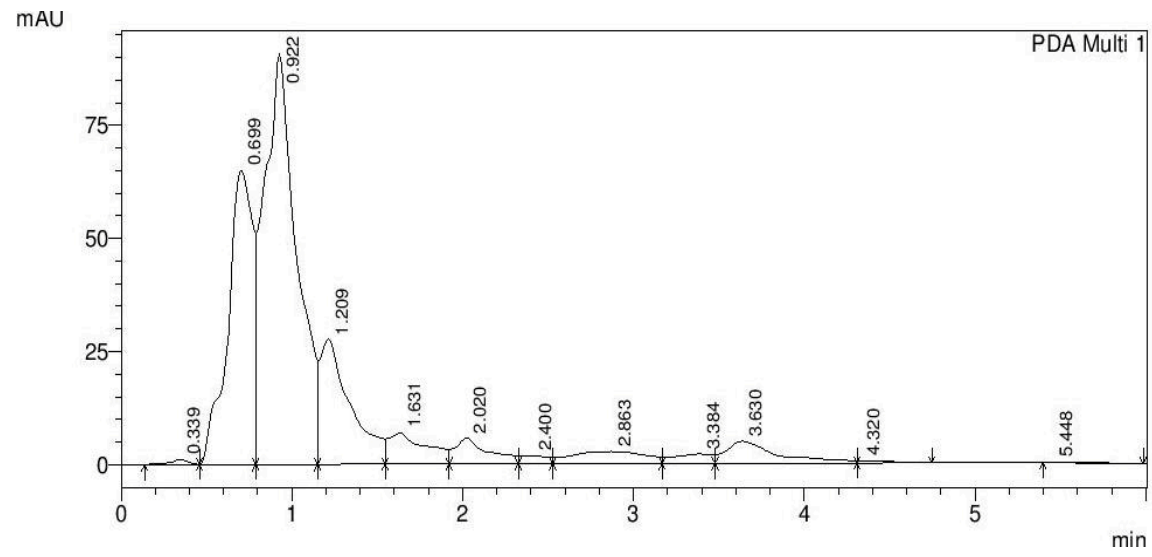

Fig. 8: Chromatogram for ESC and FLU

\section{CONCLUSION}

This article explains a simple, rapid and cost-effective method for the simultaneous quantification of ESC and FLU concentrations in plasma using HPLC coupled with PDA detector. Additionally, the method involves a simple and novel liquid phase extraction technique to process the bio-analytical samples. The ease of the method makes it more appropriate for regular therapeutic drug monitoring.

\section{ACKNOWLEDGEMENT}

The authors also thankful to Shangri-La industries [P], Sikkim for providing the gift samples of drugs ESC and FLU, respectively.

\section{FUNDING}

Nil

\section{AUTHORS CONTRIBUTIONS}

All the authors have contributed equally.

\section{CONFLICTS OF INTERESTS}

The authors declare that there are no conflicts of interest.

\section{REFERENCES}

1. Indian pharmacopoeia. Vol. II. The government of India, Ministry of health and Family Welfare. The Indian pharmacopoeia commission, Ghaziabad; 2014. p. 1685.

2. Indian pharmacopoeia. Vol. II. The government of India, Ministry of Health and Family Welfare. The Indian pharmacopoeia commission. Ghaziabad; 2014. p. 1797.

3. Rajendra B Kakde, Dinesh D Satone, Kamalesh K Gadapayale, Megha G Kakde. Stability indicating RP-HPLC method for the simultaneous determination of escitalopram oxalate and clonazepam. J Chromatogr Sci 2013;51:490-5.

4. Prasenjit Mondal, Santhosh B, Sobha Rani Satla, Ramakrishna Raparla. A new validated simultaneous RP-HPLC method for estimation of escitalopram oxalate and etizolam in bulk and tablet dosage form. Der Pharma Chemica 2013;5:26-32.

5. Kanji Fatema, Zakiur Rahman, Subrata Kumar, Biswas, Suraia Aktar. Development UV spectroscopic method for nefopam and escitalopram as INN drugs in the tablet dosage form. S J Pharm Sci 2010;3:4-10.

6. Usmangani K, Chhalotiya Kashyap, K Bhatt, Dimal A Shah, Gautam R Chauhan, Sunil L Baldania. Development of LC method for the simultaneous determination of antidepressant drug combination melitracen hydrochloride and flupentixol dihydrochloride in their combined dosage form. Chromatogr Res Int 2011;1-6. https://doi.org/10.4061/2011/632820

7. Syed Muddasir Hussain, Mohammed Aqeel, Syed Ajman Hussain. Validated spectrometric method for simultaneous estimation of flupentixol and melitracen in the combined dosage form. Int J Pharm Tech Res 2013;5:1493-7.

8. Mohd Yunus HH, Siddiqui, Paramdeep Bagga, Md Ahmad Ali, Kuldeep Singh. A simple UV spectrometric method for the determination of flupentixol dihydrochloride in bulk pharmaceutical formulation. IJPSR 2011;2:2152-5.

9. Akhil Nagar, Naresh N Chugh. Simultaneous estimation method development as an analytical method for flupentixol dihydrochloride and melitracen hydrochloride from their combined pharmaceutical dosage forms by RP-HPLC. Pharma Innovation J 2015;4:81-6.

10. El-Enany N, Belal F, El-Brashy A, El-Bahy N. Spectrofluorimetric determination of flupentixol dihydrochloride and quetiapine in pharmaceutical preparations and spiked human plasma via oxidation with cerium [IV]. ACAIJ 2009;8:325-33.

11. Damor Sheetal Ben Nareshkumar, Darpini Patel. Stability indicating chromatographic method development and validation for the simultaneous estimation of escitalopram oxalate and flupentixol in its pharmaceutical dosage form by HPLC. WJPR 2017;6:549-66.

12. Supriya T, Naresh D, Vijaya Kumar G, Haneer MA. Stability indicating RP-HPLC method development and validation for simultaneous estimation of escitalopram and flupentixol pure and marketed formulation. Asian J Pharm Res 2018;8:4-10.

13. Shivani C Patel, Dilip G Maheshwari. Development and validation of UV spectrometric and HPLC method for estimation of escitalopram oxalate and flupentixol dihydrochloride in combined dosage form. AJPTI 2016;4:59-70.

14. Singh Pinki, Dhara Patel, Dhananjay Mesbram, Sharav Desai. First-order spectrophotometric method for simultaneous estimation of escitalopram oxalate and flupentixol dihydrochloride in the pharmaceutical dosage form. IAJPR 2016;6:4544-53.

15. International Conference on Harmonization [ICH Q2B]. Guidelines on Validation of Analytical procedures: Text and Methodology. International Conference on Harmonization IFPMA, Geneva, Switzerland; 2005. 